\title{
Lymphoma TAA-specific Cytotoxic T Lymphocytes
}

National Cancer Institute

\section{Source}

National Cancer Institute. Lymphoma TAA-specific Cytotoxic T Lymphocytes. NCI

Thesaurus. Code C96736.

A population of autologous cytotoxic T lymphocytes (CT LS) with potential

immunomodulating and antitumor activities. White blood cells are grown ex-vivo and are exposed to dendritic cells (DCs) loaded with lymphoma tumor associated antigens

(TAAs); the TAA-specific CT Ls are further expanded ex-vivo before being introduced into the patient. Upon infusion with TAA-specific CT Ls, these CT Ls may help activate tumorspecific $C T L$ responses in the patient, thereby specifically killing TAA-expressing cancer cells and eventually inhibiting tumor cell proliferation. 